\title{
NONLINEAR GAUGE REALIZATION OF SPACETIME SYMMETRIES INCLUDING TRANSLATIONS
}

\author{
by \\ J. Julve, A. Lpez-Pinto, A. Tiemblo and R. Tresguerres \\ IMAFF, Consejo Superior de Investigaciones Cientficas, \\ Serrano 123, Madrid 28006, Spain
}

\begin{abstract}
We present a general scheme for the nonlinear gauge realizations of spacetime groups on coset spaces of the groups considered. In order to show the relevance of the method for the rigorous treatment of the translations in gravitational gauge theories, we apply it in particular to the affine group. This is an illustration of the family of spacetime symmetries having the form of a semidirect product $H \otimes T$, where $H$ is the stability subgroup and $T$ are the translations. The translational component of the connection behaves like a true tensor under $H$ when coset realizations are involved.
\end{abstract}

\section{Introduction}

General Relativity has a geometrical setting. It was originally formulated on a Riemannian space, in terms of coordinates of spacetime and a metric tensor defined

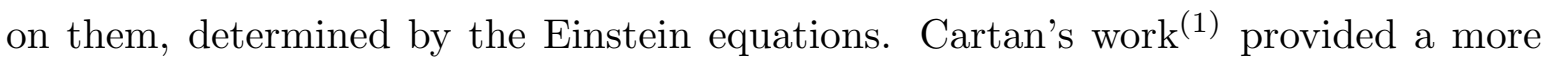
suitable, coordinate-independent, framework for the geometrical approach to theories of gravity. It is also defined on an n-dimensional manifold given a priori as an unavoidable mathematical tool, but without observable physical meaning. There, vielbeins $e_{\alpha}:=e_{\alpha}{ }^{i} \partial_{i}$ are introduced to represent local frames. Parallel transport of the reference frames is achieved by means of $1-$ form connections $\Gamma_{\alpha}{ }^{\beta}$. From the vector bases $e_{\alpha}$, coframes or bases of 1 -forms $\vartheta^{\alpha}:=e^{\alpha}{ }_{i} d x^{i}$ are defined, which are dual to the frames with respect to the interior product $\left.e_{\alpha}\right\rfloor \vartheta^{\beta}=\delta_{\alpha}^{\beta}$. The coframes thus play a central role in the geometrical formulation.

The geometrical interpretation of gravity is an essential feature of the theory. Indeed, other dynamical theories, namely the Yang-Mills theories of the remaining interactions, are necesarily defined on the geometrical background established by the 
gravitational theory. However, a unified scheme of all interactions seems to require to treat all of them on the same basis, namely as gauge theories. The first attempt to describe gravity as a dynamical theory of a local spacetime group was the work of Utiyama ${ }^{(2)}$, where just the Lorentz group was gauged, while the tetrads $\vartheta^{\alpha}$ were introduced a priori into the model as operators of reference frame changes. The coordinates were given a priori too. Later work was done to describe the tetrads as related to the gauge potentials of some spacetime symmetry group ${ }^{(3)(4)}$, in particular to those of the translations. Following this spirit, the Poincar group was introduced as the symmetry to be gauged. Wider groups are found in the literature ${ }^{(5)}$, which have the Poincar group as a subgroup. However, several problems appear when translations, as constitutive part of the spacetime gauge group, are present. The physical interpretation of the corresponding local transformations becomes dubitious, since their gauge fields do not have any geometrical meaning. The main difficulty is that one cannot identify them with the tetrads, which are covectors and lack the inhomogeneous term under gauge transformations. Some attempts were made to clarify the link between the translational connections and the true coframes. In the early works of Utiyama, Sciama and Kibble ${ }^{(2)(3)}$, the role of the translations was played by general coordinate transformations. Hehl et al. ${ }^{(4)(6)}$ proposed an improved approach, based on the Poincar group actively interpreted, regarding the gauged translations as represented by parallel transport. They substituted the usual translational generators by covariant derivatives. The price one has to pay is that the translations no longer constitute an Abelian group.

Several authors ${ }^{(7)}$ recognized that the reformulation of the dynamical gauge theory of gravity in terms of its standard geometrical structure, and vice versa, can only be realized by introducing extra nondynamical degrees of freedom in the theory. In the context of Poincar gauge theories, Grignani and Nardelli called them the Poincar coordinates $\xi^{\alpha}$. The authors considered them as Higgs-type fields which transform as vectors under gauge transformations of the Poincar group. The Lorentz group is the residual gauge group which is left invariant by the particular choice $\xi^{\alpha}=0$, called by the authors the physical gauge, because only in this case the components of the gauge potential along the Poincar generators become the physical coframe and spin connection respectively. But this restriction to the framework of a Lorentz gauge theory of gravity is not the only one which makes physical sense. In general as shown by Mielke et al. ${ }^{(8)}$, introducing a vector-valued zero form $\xi^{\alpha}$ which transforms as a Poincar (resp. as an affine) vector, and assuming that the relationship between the tetrads and the linear translational gauge fields $\stackrel{(T)}{\Gamma^{\alpha}}$ is given by

$$
\vartheta^{\alpha}:=D \xi^{\alpha}+\stackrel{(T)}{\Gamma^{\alpha}}
$$

the right transformation properties are guaranteed. Hayashi et al. ${ }^{(9)}$ considered earlier a simplified version of this definition of the tetrads. 
One of the goals of the present paper is to explain the origin of the coordinates $\xi^{\alpha}$ mentioned above as coset parameters which, as we will show below, come out from the nonlinear approach to the gauge theory of spacetime groups. Indeed, in the global limit, they become indistinguishable from Cartesian coordinates via the identification $\xi^{\alpha}=\delta_{i}^{\alpha} x^{i}$. They turn out to be an essential element in the definition of the nonlinear connection. We will show that the term $D \xi^{\alpha}$ added to the translational connection to construct the coframe, see (1.1), is a necessary contribution arising from the nonlinear realization of spacetime gauge symmetries in general.

We propose a scheme in which the coframes turn out to be nonlinear connections associated to the generators of the coset, with the right tensorial transformation properties. The translational connection does not appear as an independent object of the theory, since the translations are realized nonlineary, so that the translational covariance is present but does not become explicitely apparent. Only the particular combination (1.1) of the translational gauge fields and the coset parameters occurs, playing the role of the coframes. The whole geometrical setting arises as the result of gauging the nonlinear realization of the group in a coset space, without making recourse to any a priori structure other than the basic symmetry group. Indeed, nonlinear group realizations allow to interpret a quotient space of the whole group space as the very spacetime manifold, as noticed by several authors ${ }^{(10)}$. The coset parameters are interpreted as spacetime coordinates. The coordinate-independent formulation of the theory in terms of differential forms thus shows that the theory is independent of the coset parametrization. In our approach, the translational generators obey the usual commutation relations.

\section{Nonlinear realization of spacetime groups}

The coset representation introduced by Coleman et al. ${ }^{(11)}$ in the context of phenomenological Lagrangians is based on the non linear action of a group on itself. More precisely, the group acts on the cosets defined with respect to a subgroup $H$ which classifies the fields of the theory. The coset parameters play the role of the spacetime manifold. The explicit gauge covariance with respect to the classification subgroup is maintained. The technique, initially proposed to treat internal symmetries, was soon extended to spacetime symmetries. In fact, several attempts were made to apply the nonlinear realizations to gravity. Isham, Salam and Strathdee ${ }^{(12)}$ considered the nonlinear action of $G L(4, R)$, taking the Lorentz group as classification subgroup, see below, and Borisov and Ogievetski(13) proposed to require covariance under simultaneous nonlinear realizations of the affine and the conformal groups. But none of these works solved the main problem of explaining the relationship between the tetrads and the translational gauge fields since they only considered the global group action. Instead, a symmetric tensor constructed from the parameters of the symmetric affine transformations was identified as the "vierbein field". Stelle and West ${ }^{(14)}$ investigated the nonlinear realization allowed by the spontaneous symmetry breaking of $\mathrm{SO}(3,2)$ down to $\mathrm{SO}(3,1)$. Pseudotranslations were defined from the broken 
generators of $\mathrm{SO}(3,2)$. Their parameters, i.e. the nondynamical $\mathrm{SO}(3,2)$ vector fields $\xi^{\alpha}$ constrained to take their values in an internal anti-de Sitter space, were identified as the Goldstone fields associated to the symmetry breaking, not as coordinates. Chang and Mansouri ${ }^{(15)}$ made use of the general nonlinear approach as we do, but they did not emphasize its relevance for the gauge teatment of the translations and thus for the link between tetrads and translational gauge fields. They also introduced an auxiliary coordinate manifold alien to the group. Volkov and Soroka ${ }^{(10)}$ considered a nonlinear realization of the Poincar group in the context of the spontaneous breakdown of supersymmetry. They identified the coset parameters with the points of spacetime itself. Lord $^{(10)}$ interpreted the gauge generalization of a spacetime group $G$ in the language of fiber bundles as a gauge generalization of the stability subgroup, together with diffeomorphisms, following Hehl et al. ${ }^{(5)}$. The translations thus loose their Abelian character. According to him, the gauge potentials on the coset space are the pullbacks of the connection on $G$, while we define them as the generalized Maurer-Cartan 1-form given in eq.(3.4).

Our treatment resembles more closely that of Chang and Mansouri(15), but we identify the coset space with spacetime itself, as Lord and others ${ }^{(10)}$ do. We claim that the coset parameters do not play the role of any kind of field, but they must be considered as the coordinates themselves, as we have mentioned before.

Here we will outline the basic nonlinear machinery briefly. Let $G=\{g\}$ be a connected, semisimple Lie group, and $H=\{h\}$ a subgroup of $G$. We assume that linear representations $\rho(h)$ of the classification subgroup $H$ exist, acting on functions $\psi$ belonging to a representation space of $H$. One defines the action of $G$ on the coset space $G / H$. The "points" of $G / H$ are equivalence classes of the form $c H$, with $c \epsilon(G-H)$. Because we are dealing with Lie groups, the elements of $G / H$ are labeled by continuous parameters, say $\xi$. We choose the coset indicators $c(\xi)$ parametrized by $\xi$ as the representatives of the points of $G / H$. As we will see, these coset parameters will play the role of a kind of coordinates. Now we let act the group elements $g \epsilon G$ on $G / H$ according to

$$
\begin{aligned}
g: G / H & \rightarrow G / H \\
c(\xi) & \rightarrow c\left(\xi^{\prime}\right),
\end{aligned}
$$

according to the general law

$$
g c(\xi)=c\left(\xi^{\prime}\right) h(\xi, g)
$$

Moreover, eq. (2.2) defines the group element $h(\xi, g) \epsilon H$ governing the behavior of the fields $\psi$ under $G$. The elements $g$ of the whole group $G$ considered in (2.2) act nonlinearly on the representation space of the classification subgroup $H$ according to

$$
\psi^{\prime}=\rho(h(\xi, g)) \psi
$$

Formally, eq.(2.3) resembles the linear action of $H$, but the nonlinearity manifests itself in general through $h(\xi, g)$ as given by eq.(2.2). Generally $h$ and therefore $\psi$ 
itself are functions of $\xi$. The action of the group is realized on the pairs $(\xi, \psi)$. It reduces to the usual linear action of $H$ when we take in particular for $g$ in (2.2) an element of $H$.

\section{Local theory}

Now we will discuss the main point of this paper. Our aim is to construct the connection suitable to define a covariant differential transforming like (2.3) under local transformations. In other words, we look for the connection associated to the nonlinear realization of $\mathrm{G}$, which will behave like a connection of the classification subgroup $H$. Even in the global case, in which $g$ does not depend on $\xi$, the appearence of $\xi$ in the transformation $h(\xi, g)$, see $(2.2)$, implies that $h$ is not a constant, and a particular kind of covariant differentials is required, namely

$$
\stackrel{o}{D} \psi:=(d+\stackrel{o}{\Gamma}) \psi
$$

where $\stackrel{\circ}{\Gamma}$ is the Maurer-Cartan connection 1 -form with values on the group algebra

$$
\stackrel{o}{\Gamma}:=c^{-1} d c
$$

transforming as

$$
\stackrel{o^{\prime}}{\Gamma}=h \stackrel{o}{\Gamma} h^{-1}+h d h^{-1}
$$

We emphasize that, when local transformations are involved, the previous scheme must be extended to include a dependence of the parameters of $g \epsilon G$ on the coset coordinates $\xi$. We then generalize (3.2) to the connection corresponding to the nonlinear realization of the group, namely

$$
\Gamma:=c^{-1} \mathcal{D} c
$$

where the covariant differential on the coset space is defined as

$$
\mathcal{D} c:=(d+\Omega) c
$$

with the ordinary linear connection $\Omega$ of the whole group $\mathrm{G}$ transforming as

$$
\Omega^{\prime}=g \Omega g^{-1}+g d g^{-1} \text {. }
$$

It is easy to prove that the nonlinear gauge field $\Gamma$ defined in (3.4) transforms as

$$
\Gamma^{\prime}=h \Gamma h^{-1}+h d h^{-1}
$$

thus allowing to write true local covariant differentials of the $\psi$ fields of the theory as

$$
\mathbf{D} \psi:=(d+\Gamma) \psi
$$


obeying the same transformation law (2.3) under the local action of $g \epsilon G$. The components of the connection $\Gamma$ have very intersting transformation properties. In fact, it is easy to read out from (3.7) that

a.- only the components of $\Gamma$ related to the generators of $H$ behave as true connections, i.e. transform inhomogeneously,

b.- the components of $\Gamma$ over the generators associated with the cosets $c$ transform as tensors with respect to the subgroup $H$ notwithstanding their nature of connections.

We notice that these properties provide us with a general scheme which reproduces the main features of the gauge versions of gravity. In it the translational components of the connection appear as pure tensors, as we are going to see in the next section.

\section{Nonlinear gauge approach to the affine group}

As a relevant example, let us consider the affine group $A(n, R)=G L(n, R) \otimes R^{n}$ in $n$ dimensions, defined as the semidirect product of the translations and the general linear transformations. The following discussion is also appliable to the Poincar group. The commutation relations read

$$
\begin{aligned}
{\left[L_{\beta}^{\alpha}, L_{\nu}^{\mu}\right] } & =i\left(\delta_{\nu}^{\alpha} L_{\beta}^{\mu}-\delta_{\beta}^{\mu} L_{\nu}^{\alpha}\right), \\
{\left[L^{\alpha}{ }_{\beta}, P_{\mu}\right] } & =i \delta_{\mu}^{\alpha} P_{\beta}, \\
{\left[P_{\alpha}, P_{\beta}\right] } & =0,
\end{aligned}
$$

where $P_{\alpha}$ are the generators of the translations, and $L^{\alpha}{ }_{\beta}$ those of the linear transformations. We will realize the group action on the coset space $A(n, R) / G L(n, R)$. We choose in particular for the cosets the following parametrization:

$$
c:=e^{-i \xi^{\alpha} P_{\alpha}},
$$

where $\xi^{\alpha}$ are the coset coordinates. As we will see below, they are equivalent to Cartan's generalized radius vector or the Poincar coordinates considered by other authors $^{(7)(8)}$. The group elements of the whole affine group $A(n, R)$ are parametrized as

$$
g=e^{i a^{\alpha} P_{\alpha}} e^{i u_{\alpha}{ }^{\beta} L^{\alpha}{ }_{\beta}},
$$

and those of the classification subgroup $G L(n, R)$ are taken to be

$$
h:=e^{i u_{\alpha}^{\prime}{ }^{\beta} L_{\beta}} .
$$

Other parametrizations which lead to equivalent results are of course possible. The fundamental eq.(2.2) defining the nonlinear group action then reads

$$
e^{i a^{\alpha} P_{\alpha}} e^{i u_{\alpha}{ }^{\beta} L^{\alpha}{ }_{\beta}} e^{-i \xi^{\alpha} P_{\alpha}}=e^{-i \xi^{\prime \alpha} P_{\alpha}} e^{i u_{\alpha}^{\prime}{ }^{\beta} L^{\alpha}{ }_{\beta}} .
$$


Repeatedly using Hausdorff's formula, the explicit expressions for the transformed coset parameter $\xi^{\prime} \alpha$ and for $u_{\alpha}^{\prime}{ }^{\beta}$, in the r.h.s. of (4.5), are calculable. After a little algebra, we get

$$
\xi^{\prime}=\left(\Lambda^{-1}\right)_{\beta}{ }^{\alpha} \xi^{\beta}-a^{\alpha} \quad, \quad u_{\alpha}^{\prime \beta}=u_{\alpha}{ }^{\beta} .
$$

In eq.(4.6) and in the following, we use the definitions

$$
\Lambda_{\beta}^{\alpha}:=e^{u_{\beta}^{\alpha}} \quad, \quad\left(\Lambda^{-1}\right)_{\beta}^{\alpha}:=e^{-u_{\beta}{ }^{\alpha}} .
$$

These matrices which stand for the regular representation of (4.4) describe the two possible actions of the group associated to covariant and contravariant characters. Thus we see from (4.6) that the coset parameters $\xi^{\alpha}$ transform as affine covectors, as postulated by other authors ${ }^{(8)}$ for Cartan's generalized radius vector. We notice that in the global case the differentials of the coordinates transform as contravariant $G L(n, R)$ vectors, as seen from (4.6), so that the distinction between covariant and contravariant tensors is already present in the scheme. Observe that for the particular choice of the coset space we are dealing with, the parameters $u_{\alpha}^{\prime}{ }^{\beta}$ of the r.h.s. of (4.5), i.e. of the parameters characterizing $h \epsilon H$, according to the general formulation (2.2), coincide with those $u_{\alpha}{ }^{\beta}$ of the parametrization of $g \epsilon G$ in the l.h.s. of (4.5). Although this result is not valid for arbitrary choices of the coset space, it simplifies things in our case since the action (2.3) of the whole affine group on arbitrary fields of a representation space of $G L(n, R)$ reduces to $\psi^{\prime}=\rho(h(g)) \psi$. Actually we have

$$
\psi^{\prime}=\rho(\Lambda) \psi
$$

with

$$
\rho(\Lambda):=e^{i u_{\alpha}{ }^{\beta} \rho\left(L^{\alpha}{ }_{\beta}\right)}
$$

being an arbitrary representation of $G L(n, R)$ transformations. Now we define the suitable connection for the nonlinear gauge realization in two steps, first introducing the ordinary linear affine connection $\Omega$ in $(3.5)$ as

$$
\Omega:=-i \Gamma^{\alpha} P_{\alpha}-i \Gamma_{\alpha}^{\beta} L_{\beta}^{\alpha}
$$

which includes the true translational potential $\stackrel{(T)}{\Gamma^{\alpha}}$ and the $G L(n, R)$ connection $\Gamma_{\alpha}^{\beta}$. The transformations (3.7) take the standard form

$$
\Gamma_{\alpha}^{\prime \beta}=\left(\Lambda^{-1}\right)_{\gamma}{ }^{\beta} \Gamma_{\delta}^{\gamma} \Lambda_{\alpha}{ }^{\delta}+\left(\Lambda^{-1}\right)_{\gamma}{ }^{\beta} d \Lambda_{\alpha}^{\gamma}
$$

and

$$
\stackrel{(T)}{\Gamma^{\prime} \alpha}=\left(\Lambda^{-1}\right)_{\beta} \alpha\left[\stackrel{(T)}{\Gamma^{\beta}}+D\left(\Lambda_{\gamma}^{\beta} a^{\gamma}\right)\right],
$$


with $D$ as the covariant differential constructed with the $G L(n, R)$ connection exclusively. Making then use of definition (3.4), we get

$$
\Gamma:=e^{i \xi^{\alpha} P_{\alpha}}(d+\Omega) e^{-i \xi^{\alpha} P_{\alpha}}=-i\left(D \xi^{\alpha}+\stackrel{(T)}{\Gamma^{\alpha}}\right) P_{\alpha}-i \Gamma_{\alpha}^{\beta} L_{\beta}^{\alpha}
$$

The components on $P_{\alpha}$ in the connection (4.13) play a crucial role in gravitational gauge theories. As we mentioned before, in spite of the fact that they arise as a constitutive part of the connection required in the nonlinear realization, they do not transform as a connection, but as a covector of the classification subgroup. Let us define them as the coframe

$$
\vartheta^{\alpha}:=D \xi^{\alpha}+\stackrel{(T)}{\Gamma^{\alpha}}
$$

According to (3.7), it transforms as a covector under $G L(n, R)$. Applying $(4.6,11,12)$, we find explicitely

$$
\vartheta^{\prime \alpha}=\left(\Lambda^{-1}\right)_{\beta}^{\alpha} \vartheta^{\beta}
$$

The coframe (4.14) provides the link between the dynamical approach and the geometrical interpretation of the formalism, since it plays the role of the basis of 1 -forms. In terms of $\vartheta^{\alpha}$, we geometrize the dynamical theory defining the vector basis $e_{\alpha}$ by means of the general relation

$$
\left.e_{\alpha}\right\rfloor \vartheta^{\beta}=\delta_{\alpha}^{\beta}
$$

The vector basis transforms as a vector, namely

$$
e_{\alpha}^{\prime}=\Lambda_{\alpha}^{\beta} e_{\beta}
$$

The general linear connection in (4.13) transforms as before, see (4.11).

Commutation of two covariant differentials (3.8) yields

$$
\mathbf{D} \wedge \mathbf{D}=-i T^{\alpha} P_{\alpha}-i R_{\alpha}{ }^{\beta} L^{\alpha}{ }_{\beta},
$$

with the torsion $T^{\alpha}$ and the curvature $R_{\alpha}{ }^{\beta}$ respectively defined as

$$
T^{\alpha}:=D \vartheta^{\alpha}
$$

and

$$
R_{\alpha}^{\beta}:=d \Gamma_{\alpha}^{\beta}+\Gamma_{\gamma}^{\beta} \wedge \Gamma_{\alpha}^{\gamma}
$$

thus showing the groupal character of the torsion as the field strength of the translations.

Finally, we notice that neither the field equations nor the Noether identities as given in the literature ${ }^{(5)}$ are modified by our nonlinear approach. The diff-invariance is still present in the theory as an essential feature, thus leading to the first Noether 
identity, and the invariance under the nonlinear local action of the spacetime group yields the second Noether identity.

\section{Final remarks}

In our scheme, the expression (4.14) reproduces the same results obtained in reference (8) without any ad hoc assumptions. We claim that the general scheme of gauge nonlinear coset realizations provides the natural framework for the gauge theories of gravity. The restriction to the Poincar group follows exactly the same lines as the treatment of the affine group and consequently we do not need to insist on.

\section{Acknowledgement}

We are very grateful to Prof. F.W. Hehl for helpful comments.

\section{REFERENCES}

[1] E. Cartan, Sur les varits connexion affine et la thorie de la relativit gnralise, Ouvres completes, Editions du C.N.R.S. (1984), Partie III. 1, pgs. 659 and 921

[2] R. Utiyama, Phys. Rev. 101 (1956) 1597

[3] T. W. B. Kibble, J. Math. Phys. 2 (1961) 212

D. W. Sciama, Rev. Mod. Phys. 36 (1964) 463 and 1103

[4] F. W. Hehl, P. von der Heyde, G. D. Kerlick and J. M. Nester, Rev. Mod. Phys. 48 (1976) 393

[5] A. Trautman, in Differential Geometry, Symposia Mathematica Vol. 12 (Academic Press, London, 1973), p. 139

A. G. Agnese and P. Calvini, Phys. Rev. D 12 (1975) 3800 and 3804

E.A. Ivanov and J. Niederle, Phys. Rev. D25 (1982) 976 and 988

D. Ivanenko and G.A. Sardanashvily, Phys. Rep. 94 (1983) 1

F. W. Hehl, J. D. McCrea, E. W. Mielke, and Y. Ne'eman Found. Phys. 19 (1989) 1075

R. D. Hecht and F. W. Hehl, Proc. 9th Italian Conf. G.R. and Grav. Phys., Capri (Napoli). R. Cianci et al.(eds.) (World Scientific, Singapore, 1991) p. 246 F.W. Hehl, J.D. McCrea, E.W. Mielke, and Y. Ne'eman, Physics Reports, to be published. 
G.A. Sardanashvily, Preprint gr-qc/9405013

[6] P. von der Heyde, Phys. Lett. 58 A (1976) 141

[7] J. Hennig and J. Nitsch, Gen. Rel. Grav. 13 (1981) 947

H.R. Pagels, Phys. Rev. D 29 (1984) 1690

T. Kawai, Gen. Rel. Grav. 18 (1986) 995

G. Grignani and G. Nardelli, Phys. Rev. D 45 (1992) 2719

[8] E. W. Mielke, J.D. McCrea, Y. Ne'eman and F.W. Hehl Phys. Rev. D 48 (1993) 673 , and references therein

[9] K. Hayashi and T. Nakano, Prog. Theor. Phys 38 (1967) 491

K. Hayashi and T. Shirafuji, Prog. Theor. Phys 64 (1980) 866 and 80 (1988) 711

[10] D.V. Volkov and V.A. Soroka, JETP Lett. 18 (1973) 312, and Theor. Math. Phys. 20 (1975) 829

E.A. Lord, Gen. Rel. Grav. 19 (1987) 983

[11] S. Coleman, J. Wess and B. Zumino, Phys. Rev. 117 (1969) 2239

C.G. Callan, S. Coleman, J. Wess and B. Zumino, Phys. Rev. 117 (1969) 2247

[12] A. Salam and J. Strathdee, Phys. Rev. 184 (1969) 1750 and 1760

C.J. Isham, A. Salam and J. Strathdee, Ann. of Phys. 62 (1971) 98

[13] A.B. Borisov and V.I. Ogievetskii, Theor. Mat. Fiz. 21 (1974) 329

[14] K.S. Stelle and P.C. West, Phys. Rev. D 21 (1980) 1466

[15] L.N. Chang and F. Mansouri, Phys. Lett. 78 B (1979) 274; Phys. Rev. D 17 (1978) 3168 